\title{
In the Eye of the Hurricane - A Qualitative Study on What Is at Stake for Close Family Members to Patients Hospitalized with COVID-19
}

\author{
Camilla Bernild $^{1}$, Malene Missel ${ }^{2,}$, , Ilkay Dagyaran ${ }^{3}$, Signe Westh Christensen ${ }^{1}$, \\ Selina Kikkenborg Berg ${ }^{1,4,5}$ \\ ${ }^{1}$ The Heart Center, Rigshospitalet, Copenhagen University Hospital, Copenhagen, Denmark \\ ${ }^{2}$ Department of Cardiothoracic Surgery, the Heart Center, Rigshospitalet, Copenhagen University Hospital, Copenhagen, Denmark \\ ${ }^{3}$ Department of Infectious Diseases, the Heart Center, Rigshospitalet, Copenhagen University Hospital, Copenhagen, Denmark \\ ${ }^{4}$ National Institute of Public Health, University of Southern Denmark, Odense, Denmark \\ ${ }^{5}$ Department of Clinical Medicine, Faculty of Health and Medical Sciences, University of Copenhagen, Copenhagen, Denmark
}

Email address:

malene.missel@regionh.dk (M. Missel)

${ }^{*}$ Corresponding author

\section{To cite this article:}

Camilla Bernild, Malene Missel, Ilkay Dagyaran, Signe Westh Christensen, Selina Kikkenborg Berg. In the Eye of the Hurricane - A Qualitative Study on What Is at Stake for Close Family Members to Patients Hospitalized with COVID-19. American Journal of Nursing Science. Vol. 10, No. 4, 2021, pp. 191-200. doi: 10.11648/j.ajns.20211004.11

Received: June 14, 2021; Accepted: June 22, 2021; Published: July 2, 2021

\begin{abstract}
COVID-19 causes patient trajectories that are sudden, unpredictable and imbedded in a worldwide panic as well as lack of medical experience and knowledge. This study aims to understand how close family members to patients hospitalized with COVID-19 are affected by the situation. Individual, in-depth interviews with twelve close family members to patients hospitalized with COVID-19 were conducted. The interviews were analyzed with a hermeneutic approach using reflexive methodology. Theoretical conceptualization ensured a critical interpretation. Three empirical themes were identified: fear and unpredictability, not being able to be there and being the "key caregiver". Family members' fear is compounded as the whole world is preoccupied with the same fear and uncertainty about COVID-19. Due to the volatile situation, a shift in coping strategies throughout the trajectory was illuminated as well as an ambivalence towards the health system's treatment regime on COVID-19 implying a total separation between the patient and their close family member. In conclusion, close family members of patients hospitalized with COVID-19 are in a vulnerable situation, characterized by a high degree of unpredictability and seriousness causing fear of losing their loved ones, as well as powerlessness due to visiting restrictions. Hospitalization with COVID-19 is an unpredictable situation, where the family members are separated from their loved ones. Family members are dependent on the communication with health care professionals. Therefore, talking to family members during COVID-19 must be prioritized and it is found necessary to develop relevant and systematic practices for communication and collaboration practices.
\end{abstract}

Keywords: 2019-nCoV, COVID-19, Coronavirus, Qualitative Study, Family, Close Family Members, Hospitalization

\section{Introduction}

The World Health Organization (WHO) declared the coronavirus (COVID-19) to be a pandemic on the $11^{\text {th }}$ of March 2020 [1]. Most Governments took drastic actions and imposed a state of lockdown [2]. Thus, everybody is affected directly or indirectly by the COVID-19 pandemic in various ways physically, socially, psychologically or economically. This article is about the psychosocial consequences of COVID-19 among close family members to patients hospitalized with the disease.

The way COVID-19 appears varies from mild cold symptoms to severe and acute respiratory distress syndrome, perhaps followed by refractory hypoxemia and shock or acute kidney 
injury - some individuals have mild to moderate course of the disease, while others have lethal outcome [3]. The period from the onset of symptoms to death range from 6 to 41 days depending on the patient's age and comorbidity status [4]. Even though most research on COVID-19 regards epidemiology, clinical symptoms and possible treatments [5], there are also some research regarding the psychological impacts of the disease. The research about the psychological consequences of virus outbreaks, points out that individuals are very affected and experience feelings like fear and helplessness $[6,7]$. Research on mental health among the general public and the impact of COVID-19 shows that the virus has a great psychological impact and causes increased levels of stress, anxiety [8] depression [9] and feelings of being horrified and helpless [2]. Patients infected with COVID-19 are also reported to feel emotionally vulnerable [10] and experience that the infection is a threat to their existence as the disease appears suddenly, is unpredictable and interferes in social relationships [11], where isolation can cause alienation for others [7].

Being ill is rarely an exclusively individual matter. Neither for the patients nor their families. Inspired by a systemic approach to the family [12] we can understand a family as a unit of people who are intertwined in each other's life-worlds and are interdependent in various ways in their everyday living. Therefore, serious illness will have an impact on the family as a unit and influences the health and wellbeing of all members of the family. This viewpoint is highly relevant during the COVID19 pandemic which is creating unique hardships and suffering among affected families, where close family members because of regulations cannot visit their seriously ill loved ones [13].

Within research about COVID-19 there is a lack of research shedding light on how close family members to persons ill from COVID-19 are affected. Since the trajectory of the disease is rather sudden, unpredictable and imbedded in a worldwide panic as well as lack of medical experience and knowledge, it is of crucial importance to gain knowledge on how close family members to patients ill with COVID-19 are affected. Research involving the perspectives of close family members improves our understanding of what they go through and may enable the development of knowledgebased family-centred supportive interventions. Hence, this study aims to understand how close family members to patients hospitalized with COVID-19 are affected.

\section{Method}

\subsection{Design}

To gain knowledge about how close family members to patients hospitalized with COVID-19 are affected by the situation, a qualitative interview study was conducted [14]. The fundamental approach to gather and interpret the empirical material is philosophical hermeneutics as formulated by Gadamer (1900-2002) in his main work Truth and Method [15].

The philosophical hermeneutics is a continuation and break with Dilthey's (1833-1911) traditional, methodical and historical hermeneutics. It is still about understanding, interpreting and applicating in a hermeneutic circle moving dialectically between wholeness and parts. In philosophical hermeneutics, understanding is linked to being-in-the-world ontologically, where the argument is that if we are to understand something, we must already be in a world and then have some understanding of it in advance. Philosophical hermeneutics offers a stance that emphasizes preunderstanding, language, historicity, tradition, and meaning in relation to understand and interpret [15].

Even though preunderstandings are embedded in language and culture, and therefore not possible to objectify, we strive to obtain a transparent way of producing, interpreting and presenting the findings. To do so we are inspired by Reflective Methodology (2009) where four interpretive levels are recommended: producing the empirical material, interpretation of the text, critical interpretation of the themes and critical self-reflection - called quadri-hermeneutics [16]. In this approach it is vital to allow an open play of reflection in the process of interpretation of the interpreting subjects and interpretation of the interpretation.

Table 1. Participants.

\begin{tabular}{lllll}
\hline Participant & Relation to patient & Age & COVID 19 themselves & Information on patient \\
\hline P1 & Husband & 64 & Yes & Wife admitted on intensive care unit \\
P2 & Daughter & 57 & No & Father discharge from a medical ward \\
P3 & Daughter & 41 & No & Father admitted on intensive care unit \\
P4 & Daughter & 46 & No & Mother discharge from a medical ward \\
P5 & Wife & 53 & Yes & Husband discharged from a medical ward \\
P6 & Daughter & 49 & No & Father admitted on intensive care unit \\
P7 & Daughter & 48 & No & Mother admitted on a medical ward \\
P8 & Brother-in-law & 63 & No & Brother's wife admitted on intensive care \\
P9 & Son & 55 & No & Father admitted on intensive care unit \\
P10 & Wife & 51 & Yes & Husband discharged from intensive care unit \\
P11 & Wife & 69 & No & Husband admitted to medical ward, dementia \\
P12 & Wife & & No & Husband admitted on intensive care unit \\
\hline
\end{tabular}

All patients admitted to intensive care units had long admissions and received invasive mechanical ventilation.

\subsection{Recruitment and Participants}

Twelve close family members to patients hospitalized with
COVID-19 from three regions in Denmark participated in the period from $31^{\text {st }}$ of March to the $7^{\text {th }}$ of May. A convenience sampling strategy was used [14] by giving out flyers to 
healthcare professionals at relevant wards in different regions of Denmark describing the study and encouraging close family members to patients hospitalized with COVID-19 to e-mail the research team, if they were interested. To reduce the spread of infection the interviews were conducted by telephone while audio recorded and transcribed subsequently. Table 1 illustrates the relation the family members have to the patients and some details on the patient situation.

\subsection{Data Collection}

Using Reflexive Methodology [16] data collection is conceived as the "first level" of interpretations, namely producing the empirical material - here interviewing close family members of patients hospitalized with COVID-19 and transcribing it into text. A semi-structured interview guide [14] supported the three interviewers in covering overall areas: I) their story about the illness trajectory, II) their thoughts and feelings throughout this, III) their experience of support from family and other networks, and IV) their perspectives on communication with healthcare professionals (HCP). Throughout the interview the participants were encouraged to talk about what they found important and follow-up questions encouraging elaboration were asked. At this level, interpretation is already going on, as both parties are engaging in a dialogue and the interviewer asks certain follow-up questions on the background of (pre)understandings about what is at stake. We were however determined to explore what was said as open as possible.

\subsection{Data Analysis}

The interpretation of the text begins during the "second level". According to Alvesson and Skoldberg [16] there are no simple, self-evident or unambiguous rules or procedures when interpreting and the quality of the interpretation relies on the researchers' ability to "point something out" that is empirically founded, interesting and meaningful at a larger scale [16]. To achieve the above, all members of the research team read the twelve interviews individually, thus reading each interview in its own right and wholeness. The team met up and interpreted every interview together, aiming to understand the underlying meanings and thus found units of meaning, that appeared strongly across the twelve narratives. At this level, units of meaning in one interview were decontextualized and put together with units of meaning from other interviews with the similar content. Thus, moving from one wholeness to smaller parts, followed by a recontextualization making new wholeness: the empirically based themes. At this level new understandings of close family members of patients hospitalized with COVID-19 narratives emerged. This level of interpretation is presented in the section of Results.

"Third level" is a critical interpretation of the themes, which were done by re-reading the context of which the units of meaning were taken from, and by contextualizing the empirical findings in relation to other research and applying relevant theories [16]. The adopted theories were chosen after the interpretation at the second level and constitute our preunderstandings at the third level of interpretation. This level of interpretation is presented in the section Theoretical Interpretation and Discussion, where the fourth level of interpretation concerning the researchers' self-reflection on their own text and claims to authority [16] is also discussed. In the following, the theoretical concepts adopted are presented.

\subsection{Theoretical Concepts}

To gain an in-depth understanding of how the life-worlds of family members were affected by having their loved one hospitalized with COVID-19 a theoretical concept of lifeworld and system as developed by the philosopher Habermas was applied [17]. The life-world is a horizon or a backdrop in a community, where individuals are orientated towards an inter-subjective understanding and where symbolic reproduction takes place through communicative action.

According to Habermas, system is a historical result of rationalization of the life-world. System consists of the market's finance system and the state's bureaucratic system. As opposed to life world and its communicative action, system is upheld by the steering media; money, power/bureaucracy and law. In Habermas' theoretical concept of the relationship between life-world and system they are to be understood as mutually dependent, where the system's bureaucratically based regulations create "standard situations", which relieve the life-world from the burden of finding the right thing to do in every situation. In this case, the system has a standardized way of dealing with COVID-19, relieving the families from the burden of having to find out how to deal with the disease themselves. The risk is, however, that these regulated standard situations suppress and distort inter-subjective understanding and communication in the life-world [17].

The use of the concepts of life-world and system enhances an interpretation highlighting how the larger society affects close family members of patients hospitalized with COVID19. To further understand how the family members are affected by having their loved one hospitalized with COVID19, the concept of the Family Systems Illness model (FSI) develop by Rolland [18] inspired the interpretations. According to FSI, it is of importance to understand that several things must be considered when families are confronted with illness [19]. These are 1) illness type; including onset, course, outcome, disability and uncertainty, 2) illness time phases; including initial crisis, chronic and terminal, and 3) components of family functioning; including organization, communication, beliefs and development. Furthermore, treatment regimes, age of onset and gender are important components when families deal with illness [18]. In the interpretation of the empirically based themes the following elements from the theory are used to further unfold what is at stake for close family members of patients hospitalized with COVID-19: "illness type", especially course and uncertainty, "components of family functioning", especially organization and communication, and "treatment regimes" in terms of how the treatment of COVID-19 
includes isolation.

In order to get a deeper understanding of how the family members deal with the uncertainty COVID-19 causes, the dual model of coping with bereavement is applied (DPM) [20]. Even though DPM is concerned with loss that has taken place, the authors highlight, that it can apply to other kinds of losses, and as the participants in this study is faced by the threat of losing their loved ones at any time, it is considered highly relevant. According to DPM there is a strong connection between the stressor (the cause), the appraisal processes (the assessment of threat), coping processes (ways of dealing with the threat) and outcome variables (mental and physical indices), and it is characteristic, that individuals dealing with bereavement shift between two orientations: loss-orientation and restoration-orientation [20].

\subsection{Ethical Considerations}

Participants received written information about the purpose of the study and their right to withdraw at any time with no consequences for their treatment. Participants provided informed oral and written consent before being interviewed and were told that interview data would be treated confidentially. The interviews were held at a time chosen by the participants, so they could choose where and when themselves. The interview touched emotional topics and many participants cried or were in other ways affected when talking about their situation. The interviewers were empathic and patient throughout the interview, and many of the participants expressed relief when their experiences and feelings were put into words. The interview as well as the following analysis focused on the participants' narratives and situation, why it is not considered an ethical problem that their narratives are about a situation including their loved ones, whom we could not ask for permission. Data is kept confidential and is reported anonymous. The study was approved by the Danish Data Protection Agency (P-2020276) and followed the recommendations of the Declaration of Helsinki II [21].

\section{Results}

Across the twelve interviews, it is pervasive that the participants are very concerned about the health of their loved ones and feel powerless in a situation that is unknown and unpredictable where they cannot be there due to visiting restrictions. Through the analysis process, we have identified three coherent themes about being a close family member to someone who is hospitalized with COVID-19: Fear and unpredictability, Not being able to be there and Being the "key caregiver". The themes are unfolded below.

\subsection{Fear and Unpredictability}

The participants express in different ways how they are plagued by widespread fear, concerns and sometimes outright anxiety. Most participants narrate about a difficult time up to the point of diagnosis, where they are afraid of the risk of
COVID-19 due to their loved ones' symptoms. Often, they go through a long period of not knowing, before finally diagnosed. When confronted with the fact that their loved ones have COVID-19, most feel scared and extremely worried. The concerns of the participants are related to their risk assessment in terms of the current situation, age and comorbidity of their loved ones. The assessment stems from their own knowledge of COVID-19 gained through the media. For them all, it is a psychologically stressful life situation. When a participant was asked about the period where his loved one has been sick with COVID-19 he says: I feel like I've gotten ten years older in the three weeks [...]. I'm going to talk to my insurance at work and then I'm going to talk to a psychologist. I'm pretty sure I need that (P 12).

As the above quote illustrates, this participant considers that the situation is so stressful for him that he needs a psychologist. Moreover, the situation is so overwhelming at the time of the interview, that he can't start up the psychologist right away. This kind of situation is common for many of the participants. They are overturned by the sudden and unexpected illness and they focus on their loved one surviving - one day at a time. One says: I am damned about it really and very very worried about him getting through this (P5). As most of the participants, this family member express being very worried about whether her loved one gets through COVID-19 or not.

Through the relatives' narratives of the process, it emerges that it has been characterized by being very volatile corresponding to drastic changes in symptoms, where it goes back and forth: Because I'm starting to stop believing in it [progress]. I'm afraid things will suddenly turn again. We have just seen that (P7). As it appears, the fluctuating trajectory means that the participant does not dare to believe that small progress is part of a larger progress as it can reverse. This uncertainty is further accentuated by the fact that HCP cannot provide certainty. A participant says: It's like two steps forward and one back you could say. And you can hear that they [the health professionals] are also groping a little bit. And what hope can they give us? It's a bit of luck and a little guesswork (P 1).

What this participant expresses as a guesswork illustrates something very particular about being a family member to a patient hospitalized with COVID-19, namely that it is a new disease where HCP have no experience they can rely on, thus helping them to create predictability.

Across the interviews there is a pattern on how participants deal with their anxiety and fear of losing their loved ones in their everyday living. A participant says:

I decided that I simply wouldn't think of it [risk of losing...] because what am I going to do? I can't even take a position on that. So what I did, I went to work [...], where I could then sit down and read some emails and reply a little and do some tasks... so you get the mind off it, because there are a lot of thoughts (P 10).

It would seem that this participant deliberately tries not to think about the risk of her loved one dying and focus on something else. Several of the participants use this strategy 
deliberately, it can either be work or to embark on practical chores. However, it also appears that the participants commute back and forth between shooting away at the thought of losing their loved ones by doing something else and then being overwhelmed by the fear of losing. Some say that they have entered a process of grief in relation to losing. Participants also talk about how they try to deal with the unpredictability in the process. One says:

If it goes wrong, if I lose him, what will my life look like? But I try to remove the idea quickly, that is, because I don't want to think negatively. Then I'd rather just think day by day and every time they [the health professionals] say that's the situation is stable and it's not going the wrong way, Then I have a hope (P 12).

It would seem that this participant tries to hold on to hope by focusing on small progress or absence of decline in a dayby-day timeline. This way of dealing with the situation is repeated in different ways across the interviewed.

Although participants are concerned that their loved ones might die from COVID-19, many of them also think about how it will work out when and if their loved ones come home from the hospital. Those of the participants whose loved ones are already at home report a happy moment, but at the same time a situation of concern. After long and severe illness, their loved ones are rebutted and often remains dependent on care, needing for example help with mobilization and nutrition assistance. The participants whose loved ones are still hospitalized think about the homecoming too, as expressed here: There are no guarantees that he will be the same as he was before [...] so I hope that I can have more indulgence and capacity to accommodate him during the time [...] when he must recover and go through rehabilitation and psychological help and all that (P12). These kinds of thoughts illustrate that even though COVID-19 is an acute disease, its' severity causes worries about long term physical and psychological effects.

Due to worrying about the condition of their loved ones, several participants furthermore express concerns about their loved ones being discharged prematurely, thereby taking responsibility for a situation they do not know if they can handle. In addition to this concern, some participants talk about that they are afraid that they or others may infect their loved ones again, as immunity is not certain, and that they are vulnerable. The uncertainty illustrates the more general concern surrounding COVID-19 and the knowledge about this disease that is still lacking.

\subsection{Not Being Able to Be There}

The participants spontaneously talk about the last time they saw their loved ones, illustrating how traumatic it is not being able to visit them in the hospital. A participant expresses it like this: it has simply been the most terrible thing in this, that you couldn't come in and visit her and be near her. I think that's been the hardest (P1). Not being able to be there is spontaneously described by many participants as one of the hardest things about the situation. The participants who have had the opportunity to communicate directly with their loved ones by phone in parts of the hospitalization have been in contact many times daily. This has given them some sort of stability and has been of great importance to the participants, even though it does not substitute physical presence. Unfortunately, most have experienced long periods where their loved ones receive invasive mechanical ventilation in intensive care unit and therefore haven't been able to speak directly with them.

There are several dimensions of "not being able to be there" that affect the participants. Most profound is the fear that their loved ones will die alone: But what struck me most it was that I find it so awful to die alone [...] It was really the hardest thing for us. Not to be allowed to see her and that she does not get the feeling that we are there (P4). As expressed in this quote it is awful to think that one's loved one should die without the presence of family members. Some participants also share their reflections on how a funeral without social contact could take place, as they have heard on the news.

The fact that they cannot be there give some of the participants feelings of guilt: Because I always promised I'd be with him if he got bad [...]. I think that was the hardest thing mentally (P5). As this participant expresses the guilt of not being there is difficult to endure - even though it is due to restriction beyond their power. Although many participants share these types of thoughts, they do fully understand that they are not allowed to visit their loved ones. It does however make them feel powerless: When we become paralyzed, things get difficult. When you can't just go in and visit her and do those things (P7). As the quote illustrates being able to "visit and do things" is important to deal with the situation. The visitation-restriction caused by COVID-19 thus creates a situation that paralyzes the participants.

A few of the participants have been allowed to visit their loved ones during hospitalization, which has been important and meaningful as it strengthens them, to be able to give their loved ones' emotional support and thus combativeness in fighting for life. A participant says about not being able to visit: She may well survive medically, but she may die emotionally from isolation (P4). The quote illustrates how important this participant finds the emotional and social dimension of her loved ones' situation. Visiting is however also encumbered with worries and discomfort. Some of the participants are afraid of becoming infected with COVID19 themselves during visiting, especially those who are physically vulnerable and therefore in risk of being ill. Others feel discomfort due to protective equipment in the meeting, which they feel makes the meeting awkward and alienating.

\subsection{Being the "Key Caregiver"}

The participants' stories testify that it is most common for them to be the "key caregiver" themselves in terms of being responsible for communication with the HCP and rest of the family, friends and the wider acquaintances. This communication often takes up a great part of participants' 
time and can consist of several telephone conversations with HCP and the nearest family, as well as emails or posts on social media, where the participants inform the network about how their loved ones are doing. Thus, the "role of relative" rests predominantly on a single person. Most talk about this role as unproblematic, as they have deliberately taken this role - some even find it almost therapeutic to be the one, who takes care of the communication. Nevertheless, support from others in the family, friends and acquaintances are highlighted as important and even crucial for the participants. One says:

Well that has perhaps been the hardest thing not that you have not been able to get a cuddle from your mother [who is hospitalized with COVID-19] and just cry or something [...] in return, our neighbors have been incredibly nice so you know have sent a text hey how are you? We're going shopping in half an hour; shall we bring something? (P 10).

As the quote shows, this participant, like most of the participants, live in isolation due to COVID-19 causing lack of physical contact. Even though the support from others is often in the form of telephone calls or help with purchases, it means a lot to them, to feel cared for, when isolated and stripped from the possibility to see their loves one.

Families, friends and network can in addition to being supportive, also cause more care tasks or be an extra burden, causing extra stress and vulnerability. For example, it may be in families where the close family member's parent is hospitalized while the other parent is at home in solitary confinement, or where it is the family member's spouse who is hospitalized and that their shared children must also be cared for. A participant says about this:

Her [the mother who is admitted at the hospital] husband has also been isolated because he's been with her. So, we can't be with him. But then I cook and go to him and hand it over [...] so I also have a role in looking after him. It's such a classic. I often end up with that, but it's hard when you're also vulnerable yourself. That you must take care of others. I also have a son who's insanely affected by it, and he's also affected by the fact that I'm sad (P7).

The quote above illustrates, that there is a special task for the participants who have children living at home, where they have to deal with the difficult situation while having to protect their children from it: There are a lot of thoughts where I stopped myself especially because I have a fifteen year old sitting here which of course is also psychically affected. It is of no use that Mom sits in the sofa and cries (P 10).

Both quotes highlight that there seem to be a kind of double-role, where the participants on the one hand, deal with concerns and fear in relation to their hospitalized loved one, and on the other have to conceal this fear and be strong in order to protect their children. A few relatives are even beaten with stigma from the outside world, either in the form of timid neighbors or outright shitstorm on Facebook to be spreaders of infection. However, it is most common for social networks in the form of family, friends and acquaintances to be a positive thing, where participants receive many inquiries and supportive words.

\section{Theoretical Interpretation and Discussion}

The analysis shows that close family members to patients hospitalized with COVID-19 are in a vulnerable and stressful situation, where unpredictability and seriousness causes fear of losing their loved ones. Furthermore, they are burdened by the fact that they are not able to be there due to visiting restriction. It causes feeling of guilt and powerlessness and gives rise to even further concerns. Finally, the analysis illuminates that the family members often have the role as "key caregivers", where support and care from other family members and friends mean a lot to them. The family members who also take care of other family members, like for instance children, can experience even further burden, as they, at the same time as they are emotionally affected by the situation, have to keep strong to protect their children. In the following the findings will be contextualized and put into perspective in discussion with the chosen theoretical concepts and other research.

A profound empirical theme is the family members' experience of fear and uncertainty. To better understand this, COVID-19 can be viewed as a specific illness type, with specifics concerning the onset, outcome, cause, disability and level of uncertainty [18]. COVID-19 often has a gradual, but rather fast development, where the "typical course of severe pathology includes the appearance of overt dyspnea 6 days after the onset of flu-like symptoms, hospitalization after a further 8 days and the need for tracheal intubation 10 days after hospitalization" [22]. Thus, it can be said it has a rather sudden onset, taking the close family members unguard. This is followed by a piercing and enclosing unpredictability, due to big differences in severity in symptoms, ranging from being asymptomatic to suffer severe respiratory failure [22]. Losing their loved ones is thus a real threat. COVID-19 is not the only disease associated with sudden onsets and some sort of unpredictability, but what is unique is the general and worldwide shaken and uncertainness about COVID-19, where everybody is afraid and engaged in the attempt of understanding and controlling the disease $[2,8,9]$.

Furthermore, COVID-19 is characterized by its fluctuated trajectory, where progress can be followed by drastic drawbacks and where these fluctuations may be critical to the chances of survival. According to the FSI model [18] the degree of uncertainty is a meta-characteristic of all conditions which colors the variables of onset, course and outcome as "families coping with highly unpredictable diseases often state [...] that it is the hardest aspect to accept and master" [18]. Our study shows that the participants try to handle the uncertainty and threat of losing by having two mental tracks open at once. One track where the thought of death is pushed away, sometimes by taking on different practical chores, and where their hopes hang on small progress in their loved one's condition. Another track where they prepare themselves for their loved one's death, where the pain of anticipated loss is strong and where progress in their loved one's condition is not celebrated, because they have experienced that it can go 
back again.

This pattern in coping with the uncertainty and threat of losing someone close, can be understood by adopting the dual model of coping with bereavement is applied (DPM) [20]. According to this theory it is normal to shift between two orientations when having to deal with bereavement or the threat of bereavement: loss-orientation, where focus is on the loss and where grief takes place, and restorationorientation, where re-thinking and re-planning life takes place [20]. The participants in this study are naturally not engaged in reorientation, as their loved ones are still here, and hopefully stay. But the analysis shows that the stressor in the shape of a sudden, uncertain and life-threatening situation for their loved ones is difficult to assess unambiguously: sometimes they believe in progress as a sign that their loved ones will make it and sometimes, they think they will lose them. This causes a shift in coping strategies, where they sometimes orientate themselves towards loss and grief and sometimes focus on small progress as signs of survival. Furthermore, they shift between engaging in their loved ones' situation, by thinking about them and communicating with the network or the HCP about them, and other times engaging in other things as for example work and practical chores helping them to think about something else.

The family members' feelings of fear and uncertainty can also be understood as part of a worldwide fear and uncertainty. Using the concept of life-world, as a common horizon connecting people through communicative action as an optic magnifies this. According to Habermas, the lifeworld can be differentiated in two spheres: "the private sphere" - or better in this context the "intimate sphere", as described in his earlier work [23] and the "public sphere". Intimate sphere covers life among people in close social relations - especially families, whereas the public sphere is about communication at a larger scale in a given community - "the talk of the town", and off cause expressed in media. With this conceptualization, close family members' intimate sphere of their life-world, where their loved one might die and family life as they know it threatened, is imbedded in the "larger" life-world, where a general fear of COVID-19 fills the public sphere in society. It can be said that close family members to patients hospitalized with COVID-19 are in "the eye of the hurricane", where the intimate sphere is engaged with the same phenomena as the public sphere, thus amplifying the seriousness of the situation.

The second theme points out that the family members are tremendously affected by the fact that they cannot be there for their loved ones. According to the FSI-model the impact of treatment regime on families coping with illness is an important dimension to consider [18]. A profound characteristic of treatment regime for COVID-19 is the social isolation, which implies far reaching visiting restrictions, causing a total separation between the patient and the close family members. The participants highlighted this matter as "the worst". Studies on close family members to critically ill patients show that it is of great importance to be able to be there, when admitted at intensive care unit [24], which underlines how drastic a treatment regime this is. Even without the separation, the family response to critical illness and admission to intensive care unit includes development of psychological distress such as anxiety, acute stress disorder, depression, and grief. In order to prevent this, inclusion in care and decision-making is found to be important [25].

When reflected in the perspective of the concepts of lifeworld and system, it can be argued that the rules of the system give rise to ambivalent consequences for the lifeworld. On the one hand the systems' rules and regulations on visiting restrictions, where the standard situation is "no visitors at hospitals" are good (and necessary), because it protects from the spread of virus and risk of death. In this way it relieves the individual family from the difficult decision on whether it is a good idea to go visit their loved ones hospitalized with COVID-19. Some of the family members also expressed, that they would be worried to actually visit their loved ones, if it was allowed. On the other hand, the forced social separation from loved ones causes great emotional suffering with feeling of powerlessness. And for some a visit might have been a crucial factor in coping better with the situation.

All the participants show great understanding of why it must be this way, at the same time as they suffer a great deal from the powerlessness, guild and further uncertainty that this causes. The general worldwide strategy about "sticking together through social distancing" is thus particularly hard for close family members to patients hospitalized with COVID-19. Even though "not being able to be there" were highlighted as "the worst" for many family members, the interpretation applying the perspective of treatment regime using the concept of system and life-world, bring forward an ambivalence, where the visitation restriction also can be seen as a relief, where the decision on whether or not it is safe to visit is not an individual matter.

The third empirical theme highlights that the families often have one "key caregiver" and that their network and family situation affect them in different ways. According to the FSI model, family function is an important aspect when families deal with illness [18]. The "key caregiver" in our study is responsible for communication with the HCP, the rest of the family, friends and wider acquaintances, and not for the actual care of their loved ones. Even though the family members do not have caring tasks, the analysis shows that they spend a lot of time on their communication activities. Most find this unproblematic - some even find it therapeutic. It is worth noticing, that taking on the role as "key caregiver" in an acute and time-limited trajectory, is quite different, than having to carry out the task for a longer period of time, as it is the case with chronic diseases [18]. However, those participants who also have a caring role for others in the family, e.g. children, do express it as a stressful burden, which other studies on informal caregivers also suggest [26]. Many studies and reports about family, illness and informal caregivers, point to a gendered digression, as it is predominantly woman, who take on the task of being the "key caregiver" - if there is no spouse, that is [27]. As most 
of the participants in our study are also women it confirms this tendency, which however cannot be generalized here due to few participants.

To gain a deeper understanding of the impact of family functioning when dealing with COVID-19, further research is necessary. In this study some aspects were not examined regarding the impact of, for example, the relation between the family member and the patient, the gender, social class, network and life phase of the family members. This was because of the small number of participants. The empirical material does, however, give the impression that there might be a difference in the intensiveness of feelings of fear and powerlessness between spouses and children, as it could seem that spouses are traumatized on a larger scale than children of an older parent. This might be due to the perspective on age and life phase as well as to whether they share everyday life together or not, but in any case it requires further investigation.

\section{Study Strength and Limitation}

This study is one of few that try to understand how family members close to patients hospitalized with COVID-19, are affected. The interview method gives insight into family members' perspective and, having a philosophicalhermeneutic ontology and epistemology, supports the analysis of the interviews towards a deeper understanding of what is at stake for close family members of patients hospitalized with COVID-19. In using a philosophical hermeneutic approach, reflection on the researchers' preunderstanding is required. Besides the historically, culturally and linguistically embedded pre-understanding, that we are not able to objectify, we had the pre-understanding that family members must feel worried about their loved one's condition and burdened by the visitation restrictions. Even though the interpretation does not contradict this, the analysis gives a deeper and a more nuanced understanding, where theoretical conceptualization serves to point out the impact of an unpredictable situation, leading to dual coping strategies, the ambivalence in terms of visiting restrictions and the specifics about being the key caregiver during isolation.

We strive to obtain a trustworthy study by making each step transparent using the four levels of interpretation as recommended in Reflexive Methodology. Analytical generalizability is sought through an abductive process, where cross empirical analyses in the second level of interpretation is combined with contextualization in relation to other research as well as theoretical consolidation of the empirical themes. During the whole process the researchers reflected on the consequences of choices made - including aspects not examined. In the philosophical hermeneutic tradition this process of interpretation is called fusion of horizons [15].

The study does, however, have several limitations. Due to the risk of transmission of COVID-19 the interviews were held over the phone, making them less personal as it is not possible to have eye contact and read body language This might give less in-depth results [28]. In using a convenience sample strategy, we got hold of the participants quickly, but at the same time risked that the family members included in the study might have mental surplus and that more vulnerable family members were excluded. Due to relatively few participants, it has not been possible to investigate the impact of the type of structural relation the family member had with their loved ones; siblings, spouses/partners, parents, children and good friends, or the quality of the emotional relationship: close, dependent, difficult etc. - even though the FSI model points to this [18].

Taking up a self-reflection on our own text and claims to authority, as recommended in Reflexive Methodology [16], requires a reflection on the selectivity of the voices represented in the text. As this study focusses on the common experiences that the participants share, it presents close family members as "a collective subject". The strength in doing so is to give voice to their shared experiences. The weakness is that there are some phenomena brought forward by the individual participant that are not described. In this way there is some selectivity in the choice of voices represented, where we take on the authority to bring forward what is found most prevalent and important in the interviews. To validate these choices empirically, all researchers involved have read and re-read the interviews alongside the drafts on interpretation. Despite the attempt to gain analytical generalization through an abductive approach, the limitations of this study in terms of the telephone interview, recruitment strategy and few participants, affect the possibility to generalize its findings to a wider population and a different context.

\section{Conclusions and Implications for Clinical Practice}

This study shows that close family members of patients hospitalized with COVID-19 are in an extremely vulnerable and stressful situation, where a high degree of unpredictability and seriousness causes fear of losing their loved ones. This sense of fear is compounded by the fact that the whole world is preoccupied with the same fear and uncertainty surrounding the disease, thus creating a fusion of the public and intimate sphere. Due to the volatile and uncertain situation, they shift in coping strategies between loss-orientation and restoration-orientation. The health system's treatment regime for COVID-19 means a total separation between the patient and their close family member, and even though the family members understand and agree with this, the forced social separation causes guilt, further uncertainty and powerlessness, and ambivalent feelings towards the visiting restrictions. The study also shows that most families choose to have one "key caregiver", who is responsible for the communication between the HCP and the rest of the family and friends and that most find it unproblematic, but those "key caregivers" who have other caring tasks in the family find it stressful.

The study points out how difficult it is to be a close family member of a patient hospitalized with COVID-19 during a pandemic outbreak with the same disease. The fact that it is a 
highly unpredictable situation, where the family members are separated from their loved ones, who during their stay at intensive care unit are not able to talk, makes the family members dependent on the communication and collaboration with HCP. Therefore, HCP must prioritize talking to family members during COVID-19 and it is necessary to develop relevant and systematic practices for the communication and collaboration practices in this regard.

\section{Competing Interest}

The authors declare that they have no competing interests.

\section{Funding}

This work was supported by The Novo Nordisk Foundation (grant number NNF20SA0062831), and the Heart Center, Rigshospitalet, Copenhagen University Hospital, Denmark.

\section{Author Contributions}

All authors conceived and contributed to the design and conduct of the study. SKB, CB and MM conducted the collection of data material and led the analysis together with SWC and ID. All authors were involved in the analysis and the writing of the manuscript. All authors contributed to the preparation of this manuscript and read and approved the manuscript.

\section{Acknowledgements}

The research team wishes to thank all those people who collaborated and participated in this study by sharing their experiences. Without them, this study would not have been possible.

\section{References}

[1] World Health Organization (WHO). Coronavirus disease (COVID-19) Situation Report - 169. 2020.

[2] El-Zoghby SM, Soltan EM, Salama HM. Impact of the COVID-19 Pandemic on Mental Health and Social Support among Adult Egyptians. J Community Health. 2020 Aug; 45 (4): 689-95.

[3] Wang W, Tang J, Wei F. Updated understanding of the outbreak of 2019 novel coronavirus $(2019-\mathrm{nCoV})$ in Wuhan, China. J Med Virol. 2020 Apr 12; 92 (4): 441-7.

[4] Sohrabi C, Alsafi Z, O'neill N, Khan M, Kerwan A, Al-Jabir $A$, et al. World Health Organization declares global emergency: A review of the 2019 novel coronavirus (COVID19). Int J Surg. 2020; 76: 71-6.

[5] Zhai $\mathrm{P}$, Ding $\mathrm{Y}$, Wu X, Long J, Zhong Y, Li Y. The epidemiology, diagnosis and treatment of COVID-19. Int J Antimicrob Agents. 2020; May; 55 (5): 105955.

[6] Sim K, Huak Chan Y, Chong PN, Chua HC, Wen Soon S.
Psychosocial and coping responses within the community health care setting towards a national outbreak of an infectious disease. J Psychosom Res. 2010 Feb; 68 (2): 195-202.

[7] Missel M, Bernild C, Westh Christensen S, Dagyaran I, Kikkenborg Berg S. The marked body - a qualitative study on survivors embodied experiences of a COVID-19 illness trajectory. Scand J Caring Sci. 2021; Mar 18. doi: $10.1111 /$ scs. 12975 .

[8] Roy D, Tripathy S, Kar SK, Sharma N, Verma SK, Kaushal V. Study of knowledge, attitude, anxiety \&amp; perceived mental healthcare need in Indian population during COVID-19 pandemic. Asian J Psychiatr. 2020 Jun; 51: 102083.

[9] Mazza C, Ricci E, Biondi S, Colasanti M, Ferracuti S, Napoli C, et al. A Nationwide Survey of Psychological Distress among Italian People during the COVID-19 Pandemic: Immediate Psychological Responses and Associated Factors. Int J Environ Res Public Health. 2020 May; 17 (9): 3165.

[10] Lima CKT, Carvalho PM de M, Lima I de AAS, Nunes JVA de O, Saraiva JS, de Souza RI, et al. The emotional impact of Coronavirus 2019-nCoV (new Coronavirus disease). Psychiatry Res. 2020 May; 287: 112915.

[11] Missel M, Bernild C, Christensen SW, Dagyaran I, Berg SK. It's Not Just a Virus! Lived Experiences of People Diagnosed With COVID-19 Infection in Denmark. Qual Health Res. 2021 Feb; 104973232199036

[12] Wright, L. M. \& Leathey M. Nurses and families: A guide to family assessment and intervention. 6th ed. F. A. Davis Company; 2013.

[13] Luttik ML et. a. The COVID-19 Pandemic: A Family Affair. J Fam Nurs. 2020 May; 26 (2): 87-9.

[14] Polit DF, Beck CT. Nursing research: generating and assessing evidence for nursing practice. 11th ed. Wolters Kluwer; 2020.

[15] Gadamer H-G. Truth and Method. London: Sheed \& Ward; 1989.

[16] Alvesson M, Skoldberg K. Reflexive Methodology - New Vistas for Qualitative Research. 2nd ed. SAGE Publications; 2009.

[17] Habermas J. The Theory of Communicative Action - The Critique of Functionalist Reason. 5th ed. Beacon Press; 2004.

[18] Rolland JS. Helping Couples and Families Navigate Illness and Disability - An Integrated Approach. first edit. New York \& London: The Guildford Press; 2018.

[19] Rolland JS. Parental illness and disability: A family systems framework. J Fam Ther. 1999; 21 (3): 242-66.

[20] Stroebe M, Schut H. The Dual Process Model of Coping with Bereavement: A Decade on. OMEGA - J Death Dying. 2010 Dec; 61 (4): 273-89.

[21] World Medical Association Declaration of Helsinki. JAMA. 2013 Nov; 310 (20): 2191.

[22] Pascarella G, Strumia A, Piliego C, Bruno F, Del Buono R, Costa F, et al. COVID-19 diagnosis and management: a comprehensive review. J Intern Med. 2020 May 13; joim. 13091.

[23] Habermas J. The Structural Transformation of the Public Sphere: An Inquiry into a category of Bourgeois Society. Cambridge: Polity Press; 1989. 
[24] Dale CM, Carbone S, Istanboulian L, Fraser I, Cameron JI, Herridge MS, et al. Support needs and health-related quality of life of family caregivers of patients requiring prolonged mechanical ventilation and admission to a specialised weaning centre: A qualitative longitudinal interview study. Intensive Crit Care Nurs. 2020.

[25] Davidson JE, Jones C, Bienvenu OJ. Family response to critical illness: Postintensive care syndrome-family. Critical Care Medicine. 2012.
[26] Embracing Carers. Embracing the Critical Role of Caregivers Around the World. 2017.

[27] European Institute for Gender Equality. Gender Equality Index 2019. Work-life balance Informal care of older people, people with disabilities and long-term care services. 2019.

[28] Davies L, LeClair KL, Bagley P, Blunt H, Hinton L, Ryan S, et al. Face-to-Face Compared With Online Collected Accounts of Health and Illness Experiences: A Scoping Review. Qual Health Res. 2020 Nov; 30 (13): 2092-102. 